\title{
Perspective: Is It Time to Rename MSC (Mesenchymal Stem Cells/Medicinal Signaling Cells) with a Name that Reflects Their Combined In Vivo Functions and Their In Vitro Abilities?-Possibly "Pluripotent Mesenchymal Regulatory Cells (PMRC)”
}

\author{
David A. Hart $1,2,3$
}

${ }^{1}$ Department of Surgery and Faculty of Kinesiology, University of Calgary, Calgary, Alberta Canada; ${ }^{2}$ McCaig Institute for Bone \& Joint Health, University of Calgary, Calgary, Alberta Canada; ${ }^{3}$ Bone \& Joint Health Strategic Clinical Network, Alberta Health Services, Edmonton, Alberta Canada

Correspondence to: David A. Hart, hartd@ucalgary.ca

Keywords: Mesenchymal Stem Cells, Medicinal Signaling Cells, Pluripotent Cells, Pluripotent Mesenchymal Regulatory Cells

Received: August 1,2021 Accepted: August 28, $2021 \quad$ Published: August 31, 2021

Copyright $\odot 2021$ by author(s) and Scientific Research Publishing Inc.

This work is licensed under the Creative Commons Attribution International License (CC BY 4.0).

http://creativecommons.org/licenses/by/4.0/

\section{(c) (i) Open Access}

\section{ABSTRACT}

Over 30 years ago, it was reported by Caplan that cells could be found in various adult tissues and fluids of a variety of species that could be induced in vitro to progress towards lineages such as chondrogenesis, osteogenesis and adipogenesis with different "cocktails" of reagents. These cells were called Mesenchymal Stem Cells (MSC) to reflect this pluripotency. After 30 years of intense research effort to directly use such cells for the repair or regeneration of damaged or injured tissues, the effort has met with limited in vivo success, but their use for in vitro tissue engineering has met with some success. This failure to live up to expectations for in vivo differentiation success has led Caplan to recently rename these cells Medicinal Signaling Cells (MSC) to reflect other abilities of these cells to secrete mediators and release exosomes containing biologically active molecules that can influence their neighboring cells in a paracrine manner. However, neither of these names completely captures the combined apparent in vivo functioning of MSC and their in vitro abilities to exhibit pluripotent behavior. Thus, it is suggested, based on the attributes of these cells and their tissue and clonal heterogeneity, that an alternative name be applied to these cells and they be described as Pluripotent Mesenchymal Regulatory Cells (PMRC). This name reflects their regulatory function as pericytes in tissues, as well as their well-known immunoregulatory activity when injected into the intra-articular space and their influence on activities such as 


\section{wound healing. It also reflects their ability to differentiate along several different lineages to facilitate tissue engineering approaches for tissue repair.}

\section{INTRODUCTION}

For the past $>30$ years since mesenchymal stem cells (MSC) were reported by Caplan's group [1-3], a large number of studies have attempted to characterize these cells from a variety of adult tissues in order to assess their potential to repair and regenerate injured and damaged tissues. Cells fitting the description of MSC have been isolated from virtually all tissues [4]. The original in vitro characteristics of MSC as defined by Caplan's group [1-3] were adherence to plastic, expression of a subset of cell surface antigens and not others, and they could be induced to differentiate towards different cell lineages including chondrocytes, bone cells and adipocytes. The main sources of cells that have been used in studies to assess repair and regeneration potential of these cells are bone marrow and adipose tissues, but others have used cells from synovium, synovial fluid, Wharton's jelly, cord blood, placenta, and muscle, to name a few examples. In vivo studies have been mainly focused on using autologous cells, but others using MSC from tissues such as Wharton's jelly and placenta have been focused on allogeneic applications. Some MSC can be found circulating in the blood, and their concentration in blood declines with age.

It has been noted that MSC from most tissues or fluids are very heterogeneous [5-8], and some cells appear to have unique epigenetic signatures [9-11], as well as carbohydrate signatures as characterized by lectins $[7,12]$. Interestingly, MSC from bone marrow appear to preferentially respond to osteogenic stimuli, while MSC from synovium respond well to chondrogenic stimuli [5] in a pig model and thus, different locations may reflect the needs of such different biological and biomechanical environments.

While some successes using MSC in vivo for tissue engineering based repair of cartilage defects in humans $[13,14]$ and pig models $[15,16]$ have been noted, there have also been a number of endeavors for other applications such as for genitourinary tissues [17, 18] and solid organs [19] that have been more challenging, but progress is being made. Thus, the myriad of studies and significant effort to develop the perceived stem cell potential of adult MSC has been very challenging, but some successes have been noted with in vitro tissue engineered constructs in contrast to more limited successes following injection of free cells either systemically or into a localized site.

This lack of success in achieving the expectations that accompanied the initial reports of Mesenchymal Stem Cells, led Caplan [20,21] to suggest that perhaps MSC should not be referred to as stem cells but to the designation of them as Medicinal Signaling Cells reflecting their primary role in tissues to foster the integrity of cells in an organ via release of chemical mediators and exosomes containing mediators and other important regulatory molecules such as miRNA $[22,23]$. However, these mediator-based abilities do not preclude the potential of the tissue-associated MSC to use their in vitro pluripotent capabilities to also differentiate into lineage-specific cells for tissue engineering purposes as well. While this concept of MSC being a Medicinal Signaling Cell is intriguing and the development of the name is a somewhat logical response to the need to perhaps revisit the original expectations of using injections of undifferentiated Mesenchymal Stem Cells in vivo, this modified name does not completely capture the characteristics of the cells in vitro and in vivo. Furthermore, one cannot dismiss the option that there are multiple subsets of MSC that serve different functions in different locations at different times in the growth, maturation and senescence of a host such as a human.

Thus, it is likely that neither the term Medicinal Signaling Cell or Mesenchymal Stem Cell capture the essence of the functions and abilities of these cells. While retaining the acronym of MSC was an interesting idea from a literature perspective, perhaps it is time to consider a different name to capture the nature of the roles and abilities of the cells more accurately. This would not be the first time that the research community has had to revisit names to reflect advances in knowledge and to make more sense of what was a confusing literature. Back in the 1970s, the literature was full of mediators that were defined by the assays used to detect them. To avoid further confusion and to reconcile different assays defining the same mole- 
cule, the family of Interleukins was decided upon via cooperation within the research community. While the current circumstances with "MSC" may not be on the same scale as the interleukins, there is definitely room for better naming of what we call MSC, particularly going forward to help organize and categorize future developments in the field.

\section{IN VIVO FUNCTIONS VS IN VITRO ABILITIES OF “MSC"}

Based on the original reports of Caplan [1-3], when MSC were exposed to a stochastically defined "cocktail" of mediators and media conditions they could differentiate into a number of cell lineages. Each lineage required its own "cocktail" and conditions that result in differentiation towards specific lineages such as the chondrogenic, osteogenic or adipose lineages. In contrast, when free "MSC" were expanded in vitro and then injected into a localized environment such as the intraarticular environment of the knee or systemically via blood, the treatments did not lead to either the prolonged localization of the cells to an area of injured tissue or accelerated repair of the tissue. Thus, there was a conundrum that in vitro the "MSC" could be induced to exhibit lineage-specific pluripotency, but in vivo this potential was not overtly evident even when millions of cells were injected.

While some of this lack of localization is likely due to a lack of understanding of what recognition systems "MSC" use and how they are regulated [7], even when the recognition systems are augmented artificially there is improvement but it still does not lead to consistent large scale localization and differentiation [7]. Further studies have focused on the localization of "MSC" in tissues as pericytes that can release molecules and apparently, actively release exosomes containing mediators and regulatory molecules that can act in a paracrine manner $[22,23]$. In such a capacity, the "MSC" are functioning as regulatory cells for those cells in close proximity (paracrine influence). Whether the pluripotency of such pericyte-localized "MSC" is manifested in vivo for self-renewal purposes alone, or somehow otherwise engaged after local damage remains to be elucidated for the most part.

Finally, this dichotomy of in vitro vs in vivo functioning leads to the question of whether what are now called "MSC" would ever encounter the in vivo conditions that are required to induce differentiation in vitro towards specific lineages. Thus, for a "MSC" from BM, adipose tissue, or some other common source to encounter the complexity of mediators and signals required to induce the cells towards a chondrogenic or osteogenic lineage as defined by in vitro culture conditions would likely be a very rare event indeed. This conclusion was also discussed previously by Caplan $[20,21]$ and supported by others who also provided evidence that implanted MSC were no longer detectable in patients receiving cell transplants, and after 6 months all of the cells were of host origin [24]. Therefore, if there was a need for the MSC in the repaired/regenerated tissue, it was transient. Based on their results, this group also supported the name change suggested by Caplan [20] to Medicinal Signaling Cells [25].

Similarly, for the induction of a pluripotent stem cell (iPSC) via reversion of a somatic cell treated with a complex set of signals as defined by Yamanaka et al. [26] to become an iPSC in vivo by encounter with the necessary sequence of a defined set of circumstances to further evolve into specific lineage cells would also be very rare. Thus, the conditions developed for the in vitro differentiation of MSC and iPSC to further evolve into other lineages likely do not reflect the potential in vivo signals leading to these different lineages, or the in vitro conditions define the pluripotent potential of the cells that are never realized in vivo! The in vitro potential can still be used for tissue engineering purposes, but the expectation that the same series of events happen in vivo after inoculation may be an unreasonable expectation. Thus, unrealistic expectations from the in vitro studies may have led to a "the cart before the horse" scenario for many researchers, with a subsequent proliferation of legitimate and "shady" start-up companies, spending of billions of dollars in research funds, and disappointment for large numbers of patients with a variety of disorders. This scenario has led the FDA in the USA and Health Canada in Canada to crack down on companies making non-substantiated claims for "MSC" to cure or reverse diseases and diseased tissue via regeneration. Thus, this may be a time to rename the cells in question, question the priorities in the research agenda, and convene gatherings of diverse stakeholders to sort out the most effective directions 
forward using the knowledge gained to this point.

On balance, the above discussion regarding the exceedingly rare possibility that a MSC with pluripotency potential would encounter the conditions currently known to lead to different lineages in vivo does not eliminate the possibility that there are other mechanisms and processes available in vivo that could also lead to lineage-specific differentiation. Furthermore, the current in vitro conditions used to elaborate lineage-specific differentiation are usually performed in the absence of biomechanical stimulation, and consist of only biological factors and often with the cells on plastic. In contrast, in vivo there are not only biological signals/mediators, but also unique mechanical cues derived from the biomechanical environment as well as extracellular matrix-specific cues that could also influence differentiation processes. In vitro studies have indicated that mechanical loading can influence MSC [27, 28] and decellularized matrix can also influence these cells [29]. Therefore, these variables need to be considered when assessing the pluripotency of MSC. It is also clear that the functioning of MSC in vivo may also depend on the quality of the in vivo environment. After an injury or in the face of a chronic disease, the local in vivo environment may be "hostile" via inflammation and compromise the ability of the MSC to achieve success [30-32]. Therefore, there are a number of variables in vivo that could influence the ability of MSC to exert their pluripotency and unless controlled, interfere with success.

Aside from whether what were called Mesenchymal Stem Cells would ever encounter conditions leading to lineage-specific conditions in vivo, perhaps one should shift the focus from the use of "stem cells" to rather a focus on what the pluripotency really means and not be too hasty in removing that concept from our thinking. In other words, why would cells retain these abilities for pluripotency when perhaps a somatic cell could serve the same purpose as a Medicinal Signaling Cell? There is obvious risk involved with cells that have pluripotency abilities regarding unwanted outcomes such as potential transformation to a cancer/tumor cell. This is a possibility based on model systems [33,34]. Thus, to retain risk associated with the pluripotent abilities may indicate that these abilities serve important functions in vivo other than differentiation to unique lineages such as chondrogenesis, osteogenesis or adipogenesis. Furthermore, relevant to this issue is the finding that MSC derived from different environments exhibit preferential differentiation towards different lineages (i.e. BM towards osteogenesis and Synovium/Synovial Fluid derived towards chondrogenesis) [5]. Why this should be the case if the cells in question just serve a signaling function remains to be elucidated. Some examples of the knowledge regarding regulatory functions versus the pluripotent abilities for these cells are summarized in Table 1.

Perhaps some of the limitations of in vitro propagation of MSC to properly function when placed in vivo is related to the way the cells are cultured in vitro. That is, the in vitro conditions do not reflect very accurately the in vivo conditions that will be encountered, and thus, this compromises the ability of the pluripotency attributes to be elaborated subsequently in vivo. Improvements in in vitro culturing, such as those related to hypoxia [35,36] have led to enhanced abilities after implantation in vivo [37].

Thus, there may be subtle nuances to the pluripotency of MSC that need to be explored, and retention of the pluripotency of these cells needs to be kept in the renaming of the cells. This conclusion then raises the question of how such pluripotency could be used in vivo in the context of their functioning as pericytes. Certainly, the answer to that question should be the focus of future investigation.

\section{PLURIPOTENT MESENCHYMAL REGULATORY CELLS (PMRC)}

The name Pluripotent Mesenchymal Regulatory Cells (PMRC) is suggested to replace the term MSC for a number of reasons. First, it captures the in vitro defined abilities of the cells previously defined as MSC. Irrespective of whether this ability is manifested in vivo, such properties can be successfully subverted for tissue engineering purposes while the issue of how these properties are used in vivo gets sorted out. Second, as discussed above, in vivo evidence is mounting that such cells function mainly via release of mediators and exosomes that impact other cells in a paracrine manner. Thus, they may be considered regulatory cells. While it remains to be determined how such release of mediators and exosomes is regulated by endocrine or other systemically delivered signals, or locally generated signals (biological or mechanical), it 
Table 1. Potential regulatory functions and activities associated with the pluripotency of MSC . $^{\text {. }}$

\section{A). Regulatory Functions}

1) As pericytes in tissues: Release of tissue-specific exosomes and secretion of regulatory molecules

2) Injected as free cells into the intra-articular space to control pain in osteoarthritis:

Immunomodulatory effects with release of anti-inflammatory molecules and other regulatory mediators

3) Injected as free cells into the blood steam: Localization and functional impact at sites of renal injury

4) Homing to sites of injury: Localization to tissues such as the heart (myocardial infarction) or brain (stroke): Release mediators to assist endogenous cells to initiate repair and influence inflammation

\section{Current Limitations}

1) Understanding of why homing rate to injury/diseased sites so low

2) Knowledge of recognition systems used by MSC to perform regulatory functions

3) Why proliferation and secretory activities decline with age

B). Activities Based on Pluripotency and Proliferation

1) Self-renewal in tissues where localized as pericytes: Needed to maintain tissue integrity

2) In vitro development of tissue-engineered candidates for specific organ repair or replacement

\section{Current Limitations}

1) Inability to replicate in vitro differentiation protocols after implantation in vivo

2) Counteract the loss of MSC in vivo after implantation of in vitro generated tissue constructs

3) Failure to recapitulate in vivo conditions during development of engineered tissues in vitro

4) Propagation of cells under less than ideal conditions to reflect in vivo-improvements being made via hypoxia, serum-free, nutrients

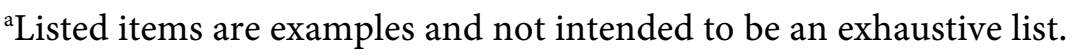

is clear that they serve a unique regulatory niche in tissues. Third, the name is broad enough to cover future developments regarding identification of functionally unique subsets of cells that may reside in different tissues and organs. Thus, the term PMRC for the cells formerly called MSC is both logical and encompassing.

\section{CONCLUSIONS}

After $>30$ years of research attempting to fulfill the expectations regarding the discovery of pluripotent cells in a variety of tissues and from a variety of species, the use of such cells for in vivo tissue repair and regeneration has been very variable. Newer information points toward these cells having a more signaling role via release of mediators and exosomes containing specific subsets of mediators. However, a role for the pluripotency of these cells has not been adequately explained and they still can be used in aspects of that role in in vitro tissue engineering for development of repair and replacement tissues. Thus, a name for these cells that capture both attributes should be retained to reflect both their regulatory functions and pluripotency abilities. The name Pluripotent Mesenchymal Regulatory Cells (PMRC) captures both areas; their regulatory function as pericytes in tissues, their immunoregulatory effects as when injected into the intra-articular space, and their influence on processes such as wound healing; as well as their abilities to differentiate along specific lineages to facilitate tissue engineering approaches for tissue repair and re- 
placement (see Table 1 for a summary of some examples of the two attributes of these cells and issues associated with each domain).

\section{ACKNOWLEDGEMENTS}

The author thanks a number of colleagues for discussions around this perspective of MSC over the past decade. These include Drs. Cy Frank (deceased), Nigel Shrive, Roman Krawetz, Arin Sen, Nori Nakamura and Rob Burnham.

\section{CONFLICTS OF INTEREST}

The author declares that he has no conflicts of interest to disclose.

\section{REFERENCES}

1. Pittenger, M.F., Discher, D.E., Peault, B.M., Phinney, D.G., et al. (2019) Mesenchymal Stem Cell Perspective: Cell Biology to Clinical Progress. NPJ Regenerative Medicine, 4, 22. https://doi.org/10.1038/s41536-019-0083-6

2. Caplan, A.I. (1990) Stem Cell Delivery Vehicle. Biomaterials, 11, 44-46.

3. Caplan, A.I. (1991) Mesenchymal Stem Cells. Journal of Orthopaedic Research, 9, 641-650. https://doi.org/10.1002/jor.1100090504

4. da Silva Meirelles, L., Chagastelles, P.C. and Nardi, N.B. (2006) Mesenchymal Stem Cells Reside in Virtually All Post-Natal Organs and Tissues. Journal of Cell Science, 119, 2204-2213. https://doi.org/10.1242/jcs.02932

5. Ando, W., Kutcher, J.J., Krawetz, R., Sen, A., et al. (2014) Clonal Analysis of Synovial Fluid Stem Cells to Characterize and Identify Stable Mesenchymal Stromal Cell/Mesenchymal Progenitor Cell Phenotypes in a Porcine Model: A Cell Source with Enhanced Commitment to the Chondrogenic Lineage. Cytotherapy, 16, 776-788. https://doi.org/10.1016/j.jcyt.2013.12.003

6. Hart, D.A. (2014) Why Mesenchymal Stem/Progenitor Cell Heterogeneity in Specific Environments? Implications for Tissue Engineering Applications Following Injury or Degeneration of Connective Tissues. Journal of Biomedical Science and Engineering, 7, 526-532. https://doi.org/10.4236/jbise.2014.78054

7. Hart, D.A. (2021) What Molecular Recognition Systems Do Mesenchymal Stem Cells/Medicinal Signaling Cells (MSC) Use to Facilitate Cell-Cell and Cell Matrix Interactions? A Review of Evidence and Options. International Journal of Molecular Sciences, 22, 8637. https://doi.org/10.3390/ijms22168637

8. McLeod, C.M. and Mauck, R.L. (2017) On the Origin and Impact of Mesenchymal Stem Cell Heterogeneity: New Insights and Emerging Tools for Single Cell Analysis. European Cells \& Materials, 34, 217-231. https://doi.org/10.22203/eCM.v034a14

9. Brown, C., McKee, C., Bakshi, S., Walker, K., et al. (2019) Mesenchymal Stem Cells: Cell Therapy and Regeneration Potential. Journal of Tissue Engineering and Regenerative Medicine, 13, 1738-1755. https://doi.org/10.1002/term.2914

10. Ng, T.T., Mak, K.H.M., Popp, C. and Ng, R.K. (2020) Murine Mesenchymal Stromal Cells Retain Biased Differentiation Plasticity towards Their Tissue of Origin. Cells, 9, 756. https://doi.org/10.3390/cells9030756

11. Wang, T., Zhang, J., Liao, L., Zhang, F. and Zhou, G. (2020) Donor Genetic Backgrounds Contribute to the Functional Heterogeneity of Stem Cells and Clinical Outcomes. Stem Cells Translational Medicine, 9, 1495-1499. https://doi.org/10.1002/sctm.20-0155

12. Talaei-Khozani, T., Aleahmad, F., Bazrafshan, A., Alibadi, E., et al. (2019) Lectin Profile Variation in Mesenchymal Cells Derived from Different Sources. Cell Tissues Organs, 208, 101-112. https://doi.org/10.1159/000505238 
13. Shimomura, K., Yasui, Y., Koisumi, K., Chijimatsu, R., et al. (2018) First-in-Human Pilot Study of Implantation of a Scaffold-Free Tissue-Engineered Construct Generated from Autologous Synovial Mesenchymal Stem Cells for Repair of Chondral Lesions. The American Journal of Sports Medicine, 46, 2384-2393. https://doi.org/10.1177/0363546518781825

14. Shimomura, K., Hamada, H., Hart, D.A., Ando, W., et al. (2021) Histological Analysis of Cartilage Defects Repaired with an Autologous Human Stem Cell Construct 48 Weeks Post-Implantation Reveals Structural Details Not Detected by T2 Mapping MRI. Cartilage. https://doi.org/10.1177/1947603521989423

15. Ando, W., Tateishi, K., Hart, D.A., Katakai, D., et al. (2007) Cartilage Repair Using an in Vitro Generated Scaffold-Free Tissue-Engineered Construct Derived from Porcine Synovial Mesenchymal Stem Cells. Biomaterials, 28, 5462-5470. https://doi.org/10.1016/j.biomaterials.2007.08.030

16. Shimomura, K., Ando, W., Tateishi, K., Nansai, N., et al. (2010) The Influence of Skeletal Maturity on Allogenic Synovial Mesenchymal Stem Cell-Based Repair of Cartilage in a Large Animal Model. Biomaterials, 31, 8004-8011. https://doi.org/10.1016/j.biomaterials.2010.07.017

17. Wang, X., Zhang, F. and Liao, L. (2021) Current Applications and Future Directions of Bioengineering Approaches for Bladder Augmentation and Reconstruction. Frontiers in Surgery, 8, Article ID: 664404. https://doi.org/10.3389/fsurg.2021.664404

18. Caneparo, C., Brownwell, D., Chabaud, S. and Bolduc, S. (2021) Genitourinary Tissue Engineering: Reconstruction and Research Models. Bioengineering (Basel), 8, 99. https://doi.org/10.3390/bioengineering8070099

19. Jorgensen, A.M., Yoo, J.J. and Atala, A. (2020) Solid Organ Bioprinting: Strategies to Achieve Organ Function. Chemical Reviews, 120, 11093-11127. https://doi.org/10.1021/acs.chemrev.0c00145

20. Caplan, A.I. (2017) Mesenchymal Stem Cells: Time to Change the Name. Stem Cells Translational Medicine, 6, 1445-1451. https://doi.org/10.1002/sctm.17-0051

21. Caplan, A.I. (2019) There Is No "Stem Cell Mess". Tissue Engineering Part B: Reviews, 25, 291-292. https://doi.org/10.1089/ten.teb.2019.0049

22. Wei, W., Ao, Q., Wang, X., Cao, Y., et al. (2021) Mesenchymal Stem Cell-Derived Exosomes: A Promising Biological Tool in Nanomedicine. Frontiers in Pharmacology, 11, Article ID: 590470. https://doi.org/10.3389/fphar.2020.590470

23. Shi, J., Zhao, Y.C., Niu, Z.F., Fan, H.J., et al. (2021) Mesenchymal Stem Cell-Derived Small Extracellular Vesicles in the Treatment of Human Diseases: Progress and Prospects. World Journal of Stem Cells, 13, 49-63. https://doi.org/10.4252/wjsc.v13.i1.49

24. de Windt, T.S., Vonk, L.A., Slaper-Cortenbach, J.M., Nizak, R., et al. (2017) Allogeneic MSCs and Recycled Autologous Chondrons Mixed in a One-Stage Cartilage Cell Transplantation: A First-in-Man Trial in 35 Patients. Stem Cells, 35, 1984-1993. https://doi.org/10.1002/stem.2657

25. de Windt, T.S., Vonk, L.A. and Saris, D.B.F. (2017) Response to: Stem Cells: Time to Change the Name! Stem Cells Translational Medicine, 6, 1747-1748. https://doi.org/10.1002/sctm.17-0120

26. Yamanaka, S. (2012) Induced Pluripotent Stem Cells: Past, Present and Future. Cell Stem Cell, 10, 678-684. https://doi.org/10.1016/j.stem.2012.05.005

27. Yong, K.W., Choi, J.R., Choi, J.Y. and Cowie, A.C. (2020) Recent Advances in Mechanically Loaded Human Mesenchymal Stem Cells for Bone Tissue Engineering. International Journal of Molecular Sciences, 21, 5816. https://doi.org/10.3390/ijms21165816

28. Ge, Y., Li, Y., Wang, Z., Li, L., et al. (2021) Effects of Mechanical Compression on Chondrogenesis of Human Synovium-Derived Mesenchymal Stem Cells in Agarose Hydrogel. Frontiers in Bioengineering and Biotechnology, 9, Article ID: 697281. https://doi.org/10.3389/fbioe.2021.697281 
29. Gentile, P., Sterodimas, A., Pizzicannella, J., Dionisi, L., et al. (2020) Systemic Review: Allogenic Use of Stromal Vascular Fraction (SVF) and Decellularized Extracellular Matrices (ECM) as Advanced Therapy Medicinal Products (ATMP) in Tissue Regeneration. International Journal of Molecular Sciences, 21, 4982. https://doi.org/10.3390/ijms21144982

30. Hart, D.A., Kydd, A.S., Frank, C.B. and Hildebrand, K.A. (2004) Tissue Repair in Rheumatoid Arthritis: Challenges and Opportunities in the Face of a Systemic Inflammatory Disease. Best Practice \& Research: Clinical Rheumatology, 18, 187-202. https://doi.org/10.1016/j.berh.2004.02.007

31. Ando, W., Heard, B., Nakamura, N., Frank, C.B. and Hart, D.A. (2012) Ovine Synovial Membrane-Derived Mesenchymal Progenitor Cells Retain the Phenotype of the Original Tissue That Was Exposed to in Vivo Inflammation: Evidence for a Suppressed Chondrogenic Differentiation Potential of the Cells. Inflammation Research, 61, 599-608. https://doi.org/10.1007/s00011-012-0450-x

32. Krawetz, R.J., Wu, Y.E., Martin, L., Rattner, J.B., et al. (2012) Synovial Fluid Progenitors Expressing CD90+ from Normal But Not Osteoarthritis Joints Undergo Chondrogenic Differentiation without Micro-Mass Culture. PLoS ONE, 7, e43616. https://doi.org/10.1371/journal.pone.0043616

33. He, X., Li, B., Shao, Y., Hsu, Y., et al. (2015) Cell Fusion between Gastric Epithelial Cells and Mesenchymal Stem Cells Results in Epithelial-to-Mesenchymal Transition and Malignant Transformation. BMC Cancer, 15, 24. https://doi.org/10.1186/s12885-015-1027-1

34. Zhang, Q., Chai, S., Wang, W., Wan, C., et al. (2019) Macrophages Activate Mesenchymal Stem Cells to Acquire Cancer-Associated Fibroblast-Like Features Resulting in Gastric Epithelial Cell Leasions and Malignant Transformation in Vitro. Oncology Letters, 17, 747-756. https://doi.org/10.3892/ol.2018.9703

35. Nikolitis, I., Nebel, S., Egger, D., Kress, S., et al. (2021) Towards Physiologic Culture Approaches to Improve Standard Cultivation of Mesenchymal Stem Cells. Cells, 10, 886. https://doi.org/10.3390/cells10040886

36. Hu, C., Wu, Z. and Li, L. (2020) Pre-Treatments Enhance the Therapeutic Effects of Mesenchymal Stem Cells in Liver Diseases. Journal of Cellular and Molecular Medicine, 24, 40-49. https://doi.org/10.1111/jcmm.14788

37. Ishiuchi, N., Nakashima, A., Doi, S., Maeda, S., et al. (2021) Serum-Free Medium and Hypoxic Preconditioning Synergistically Enhance the Therapeutic Effects of Mesenchymal Stem Cells on Experimental Renal Fibrosis. Stem Cell Research \& Therapy, 12, 472. https://doi.org/10.1186/s13287-021-02548-7 